\title{
Alternatively spliced variants of the regucalcin gene in various human normal and tumor tissues
}

\author{
TOMIYASU MURATA $^{1}$ and MASAYOSHI YAMAGUCHI ${ }^{2}$ \\ ${ }^{1}$ Department of Analytical Neurosciences, Faculty of Pharmaceutical Sciences, Meijo University, Nagoya, Japan; \\ ${ }^{2}$ Department of Hematology and Medical Oncology, Emory University School of Medicine, Atlanta, GA, USA
}

Received April 12, 2014; Accepted July 7, 2014

DOI: $10.3892 / \mathrm{ijmm} .2014 .1858$

\begin{abstract}
Regucalcin plays a pivotal role as a suppressor protein in signal transduction in various cell types. The regucalcin gene, which is localized on the $\mathrm{X}$ chromosome, consists of 7 exons and 6 introns. Decreased liver regucalcin gene expression has been suggested to play a suppressive role in the development of hepatocellular carcinogenesis in animal models. This study was undertaken to determine the changes in regucalcin gene expression in various human normal and tumor tissues, including liver, kidney, brain and lung tissues. The full-length and alternatively spliced variants of regucalcin mRNA were found to be expressed in various human tissues. This expression was suppressed in tumor tissues of hepatocellular carcinoma, kidney transitional cell carcinoma, brain malignant meningioma and lung non-small cell carcinoma. The full-length regucalcin protein was found to be highly expressed in normal human liver and kidney tissues; its expression was suppressed, however, in the liver and kidney tumor tissues. The spliced variant proteins were found to be expressed in the normal liver and kidney tissues, and decreased in the tumor tissues. Such alternative variants were not observed in the liver and kidneys of rats and mice. The alternatively spliced variants of the regucalcin gene were found to be expressed in various human normal and tumor tissues.
\end{abstract}

\section{Introduction}

Regucalcin was first identifed in 1978 as a novel calciumbinding protein that differs from calmodulin and other calcium-related proteins (1-5). The regucalcin gene ( $r g n)$ is localized on the X chromosome (6,7), and it is identified in over 15 species consisting of the regucalcin family in vertebrate

Correspondence to: Dr Masayoshi Yamaguchi, Department of Hematology and Medical Oncology, Emory University School of Medicine, 1365 C Clifton Road, NE, Atlanta, GA 30322, USA

E-mail: yamamasa1155@yahoo.co.jp

Key words: regucalcin, human tumor, alternative spliced valiant, gene expression, carcinogenesis and invertebrate species and is highly conserved in vertebrate species $(4,5)$. The organization of the rat regucalcin gene consists of 7 exons and 6 introns and several consensus regulatory elements exist upstream of the 5'-flanking region (8). Activator protein 1 (AP-1), nuclear factor 1-A1 (NF1-A1), regucalcin gene promoter region-related protein p117 (RGPR-p117), $\beta$-catenin and other factors have been found to be transcription factors in the enhancement of regucalcin gene promoter activity (5). The transcriptional activity of the regucalcin gene is enhanced through intracellular signaling factors that are mediated through the phosphorylation and dephosphorylation of nuclear protein in vitro (5). As previously demonstrated, regucalcin mRNA and protein are pronouncedly expressed in the liver and kidney cortex of rats $(9,10)$. The mRNA expression of regucalcin in the liver and kidney cortex has been shown to be stimulated by hormonal factors (including calcium, calcitonin, parathyroid hormone, insulin, estrogen and dexamethasone) (11).

Regucalcin, which plays a pivotal role as a suppressor protein in cell signal transduction, plays a multifunctional role in the regulation of various types of cells and tissues (reviewed in refs. 12-14). Regucalcin translocates from the cytoplasm to the nucleus in various cell types (15). Regucalcin has been shown to play a role in maintaining intracellular calcium homeostasis, the inhibition of various protein kinases and protein phosphatases in the cytoplasm and nucleus, and nuclear DNA and RNA synthesis $(14,15)$. Nuclear regucalcin has also been shown to regulate the gene expression of various proteins (15). Moreover, regucalcin suppresses cell proliferation and apoptotic cell death that is mediated through various signaling factors $(16,17)$. Regucalcin has been suggested to play a physiological role in maintaining cell homeostasis and function as the regulatory protein of intracellular signaling systems $(13,14)$.

There is growing evidence that regucalcin plays a pathophysiological role in metabolic disorder $(16,18,19)$. In recent years, regucalcin has been shown to be involved in carcinogenesis (reviewed in ref. 16). Regucalcin gene and protein expression has been shown to be decreased in the liver tumor tissue of rats in vivo (20). Of note, regucalcin gene expression has been demonstrated to be downregulated during the development of carcinogenesis in the chemical-treated regenerating rat liver in vivo, suggesting that regucalcin plays a suppressive role in carcinogenesis (21). Moreover, regucalcin gene and protein expression has been shown to be suppressed in cloned 
rat hepatoma H4-II-E cells in vitro (22-25). The overexpression of endogenous regucalcin has been found to suppress the enhancement of cell proliferation with fetal bovine serum in H4-II-E cells in vitro due to the inhibition of various protein kinases and phosphatases, nuclear DNA synthesis and the mRNA expression of oncogenes (22-25). Thus, regucalcin may play a suppressive role in the development of carcinogenesis. However, the findings of regucalcin gene expression in human normal and tumor tissues require further confirmation.

The present study was undertaken in order to determine whether the regucalcin gene is expressed in various human normal and tumor tissues including liver, kidney, brain and lung tissues. We found that the full-length regucalcin mRNA and its alternatively spliced variants were expressed in various human tissues.

\section{Materials and methods}

Materials. Regucalcin was isolated from rat liver as previously described (1). A polyclonal rabbit anti-regucalcin antibody was prepared as previously described (10). Other antibodies for western blot analysis were purchased from Santa Cruz Biotechnology (Santa Cruz, CA, USA). Reagents for polymerase chain reaction (PCR) were purchased from Invitrogen (Carlsbad, CA). All other reagents were purchased from Sigma-Aldrich Chemical Corp., (St. Louis, MO, USA) unless otherwise indicated.

Human cDNA and protein samples. This study was approved by the Ethics Committee of Meijo University (Nagoya, Japan). The human cDNA and protein samples, which were prepared from normal (non-tumor) and tumor tissues, were obtained from BioChain Institute, Inc. (Newark, CA, USA). In the expression analysis of regucalcin mRNA in the human normal and tumor tissues, cDNA prepared from the following tissues was used: normal liver from a 64-year-old male; normal kidney from a 46-year-old male; normal brain from a 26-year-old male; normal lung from a 41-year-old female; liver hepatocellular carcinoma from a 51-year-old male; kidney transitional cell carcinoma froma a 68-year-old male; brain malignant meningioma from a 7-year-old male; and lung non-small cell carcinoma from a 46-year-old male. In the expression analysis of regucalcin mRNA in the human brain, cDNA prepared from the following tissues was used: amygdale from a 60-year-old female, cerebral cortex from a 28 -year-old male, cerebellum from a 27 -year-old male, diencephalon from a 26-year-old male, hippocampus from a 28-year-old male, medulla oblongata from a 26-year-old male, optic nerve from a 22-year-old male, pituitary gland from a 68-year-old male and thalamus from a 22-year-old male. In the expression analysis of regucalcin protein in normal human liver and kidney, total protein lysates prepared from the following tissues were used: normal liver from a 24-year-old male and normal kidney from a 62-year-old male. In the expression analysis of regucalcin protein in human liver and kidney tumor tissues, the total protein lysates prepared from the following tissues were used: liver hepatocellular carcinoma and its adjacent normal liver tissue from a 39-year-old male; and kidney clear cell carcinoma and its adjacent normal kidney tissue from a 64-year-old male. These normal and tumor tissues used in the preparation of cDNA and protein samples were estimated by clinical diagnosis.

Detection of alternatively spliced variants. Total RNA in the non-tumor (normal) and tumor tissues from human liver, kidney, brain and lung tissues (BioChain Institute) was isolated using the guanidine thiocyanate technique. Total RNA $(11 \mu \mathrm{g})$ was primed by an oligo(dT) primer and reverse transcribed using reverse transcriptase in a $40 \mu \mathrm{l}$ final volume. The reaction was terminated by heating at $65^{\circ} \mathrm{C}$ for $10 \mathrm{~min}$. cDNA was delivered in $1 \mathrm{X}$ RT buffer (50 mM Tris- $\mathrm{Cl}, \mathrm{pH} 8.3,75 \mathrm{mM}$ $\mathrm{KCl}, 3 \mathrm{mM} \mathrm{MgCl}_{2}, 10 \mathrm{mM}$ dithiothreitol). The estimated cDNA concentration was approximately $2.5 \mathrm{ng} / \mu 1$. cDNA (2.5-5 $\mathrm{ng} / \mu \mathrm{l})$ was used as the template in polymerase chain reaction (PCR). The amount of cDNA, which was normalized to the actin mRNA levels, was used for PCR. PCR was performed using the following protocol: initial denaturation at $95^{\circ} \mathrm{C}$ for $45 \mathrm{sec}$, followed by 30 cycles of $94^{\circ} \mathrm{C}$ for $45 \mathrm{sec}, 56^{\circ} \mathrm{C}$ for $1 \mathrm{~min}$, and $72^{\circ} \mathrm{C}$ for $1 \mathrm{~min}$, with a final extension at $72^{\circ} \mathrm{C}$ for $3 \mathrm{~min}$. The primers used for the detection of human regucalcin cDNA were 5'-ATGTCTTCCATTAAGATTGAGTGTGT-3' for the sense primer and 5'-TCATCCCGCATAGGAGTAGGG AGCA-3' for the antisense primer. The amplified PCR products were visualized on a $1.8 \%$ agarose gel prepared with the addition of ethidium bromide. To determine the DNA sequences of the PCR products, amplified DNA fragments were cloned into the pGEM-T Easy cloning vector and then sequenced with an automated fluorescence DNA sequencer (3130 Genetic Analyzer; Applied Biosystems, Foster City, CA, USA) to confirm the identity of the cloned inserts. The house keeping gene, $\beta$-actin, was also amplified to normalize the cDNA content of each sample.

Western blot analysis. Total protein lysates, which were obtained from normal and tumor tissues of human liver and kidney (BioChain Institute), were used for western blot analysis. The total protein lysates were subjected to $12 \%$ SDS-polyacrylamide gel electrophoresis, and proteins on the gel were transferred to PVDF membranes. The membranes were blocked in TBS-T (20 mm Tris, $\mathrm{pH} \mathrm{7.5,} 150 \mathrm{~mm} \mathrm{NaCl}$ and $0.1 \%$ Tween-20) supplemented with $5 \%$ non-fat dry milk for $1 \mathrm{~h}$ at room temperature. The first regucalcin antibody that we previously produced against a purified rat regucalcin protein $(10)$ was diluted $(1: 1,000)$ in Can get signal immunoreaction solution 1 (Toyobo, Tokyo, Japan). The secondary antibody, an affinity purified horseradish peroxidase (HRP)conjugated goat anti-rabbit IgG (Invitrogen Corp.) was diluted $(1: 1,000)$ in Can get signal immunoreaction solution 2 (Toyobo). The membrane was probed with a rabbit polyclonal regucalcin antibody overnight at $4^{\circ} \mathrm{C}$, followed by HRP-conjugated goat anti-rabbit IgG polyclonal antibodies for $1 \mathrm{~h}$ at room temperature. Following immunoprobing with primary and secondary antibodies, the blots were washed 3 times with TBS-T. The blots were developed using ECL chemiluminescence reagent according to the manufacturer's instructions (Amersham Biosciences, Pittsburgh, PA, USA). For reprobing, the membranes were incubated in a stripping buffer (Pierce, Rockford, IL, USA) for $20 \mathrm{~min}$ and were then reprobed with anti- $\beta$-actin antibody (Santa Cruz Biotechnology) as a loading control. 

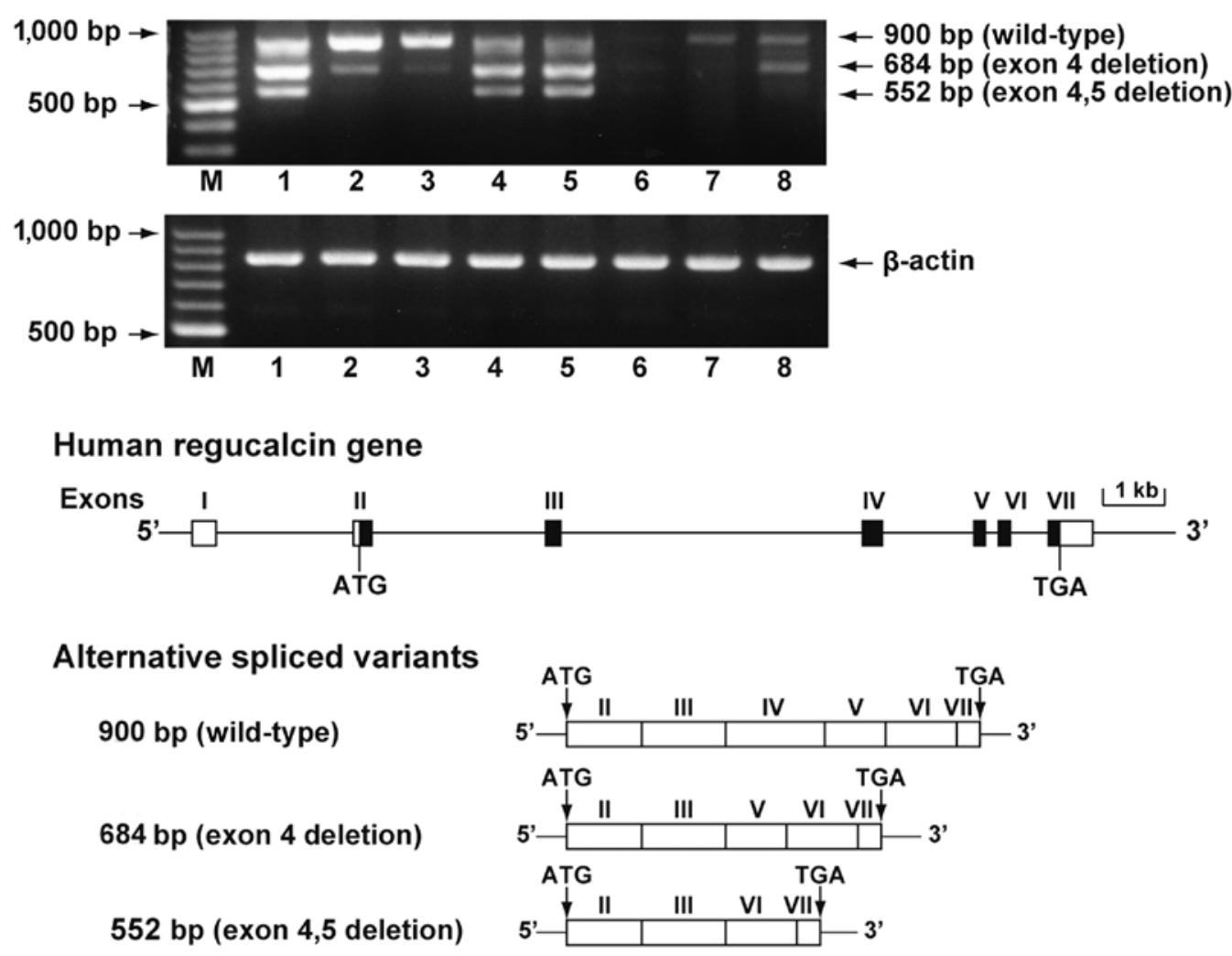

Figure 1. Expression of regucalcin mRNA in human normal and tumor tissues. The coding region of the regucalcin gene was amplified using the cDNA for total RNA obtained from human normal and tumor tissues with the method of PCR. The amplified PCR products were visualized on a $1.8 \%$ agarose gel containing ethidium bromide. Data are representative of one of 3 experiments. M, 100 base DNA-ladder; lane 1, normal liver; lane 2, normal kidney; lane 3, normal brain; lane 4, normal lung; lane 5, tumor liver (hepatocellular carcinoma); lane 6, tumor kidney (transitional cell carcinoma); lane 7, tumor brain (malignant meningioma); lane 8 , tumor lung (non-small cell carcinoma).

\section{Results}

Regucalcin mRNA expression in the normal and tumor tissues of human liver, kidney, brain and lung is shown in Fig. 1. The coding region of regucalcin gene was amplified using cDNA from total RNA obtained from the human tissues. Full-length regucalcin mRNA (900 bp) was expressed in the normal tissues of the liver, kidneys, brain and lungs; its expression was suppressed in tumor tissues, including liver hepatocellular carcinoma, kidney transitional cell carcinoma, brain malignant meningioma and lung non-small cell carcinoma with clinical diagnosis. We determined the DNA sequence of 2 PCR products derived from transcripts. The cloned PCR products consist of 684-bp fragment with exon 4 deletion and a 552-bp fragment with exon 4 and 5 deletions. These results indicated that 2 alternatively spliced variants were present in regucalcin mRNA. The 2 spliced transcripts encode the 2 predicted proteins of 227 amino acids $(\sim 25 \mathrm{kDa})$ and 183 amino acids ( 20 kDa). The 2 alternatively spliced variants were detected in normal tissues, including the liver, kidneys, brain and lungs. The expression levels of the 2 alternatively spliced variants were high in the normal liver and lung tissue. Of note, the expression of the 2 alternatively spliced variants appeared to be decreased in the tumor tissues of the liver, kidneys, brain and lungs.

Regucalcin mRNA expression in the various brain tissues obtained from healthy humans is shown in Fig. 2. Full-length regucalcin mRNA was expressed in the amygdala, cerebral cortex, cerebellum, diencephalon, hippocampus, medulla oblongata, optic nerve, pituitary and thalamus. Alternatively the spliced variants were also detected in the cerebral cortex and pituitary tissues. In other brain tissues, the expression of these variants was minimal.

The expression of regucalcin protein in the liver and kidney tissues obtained from healthy humans is shown in Fig. 3. Full-length regucalcin protein of $34 \mathrm{kDa}$ was markedly detected in the normal liver and kidney tissues. In addition, 4 weak bands with 28,25, 24 and $20 \mathrm{kDa}$ were observed in the liver tissues, and the band with $27 \mathrm{kDa}$ was found in the kidney tissue. The 2 alternatively spliced transcripts indicated in Fig. 1 encoded 2 predicated proteins of approximately 25 and $20 \mathrm{kDa}$. The liver protein bands of 25 and $20 \mathrm{kDa}$ were associated with the calculated molecular weights of the regucalcin splice variant proteins. The expression levels of 2 splice variant proteins were minimal as compared with those of the full-length protein. Furthermore, the liver protein bands of 28 and $24 \mathrm{kDa}$ and kidney protein band of $27 \mathrm{kDa}$, which were not comparable to the predicted molecular weights of the splice variant proteins, were detected at minimal levels.

The expression of regucalcin protein in the normal and tumor tissues of liver and kidney obtained from humans is shown in Fig. 4. The expression of regucalcin proteins with different molecular weight appeared to be decreased in the 


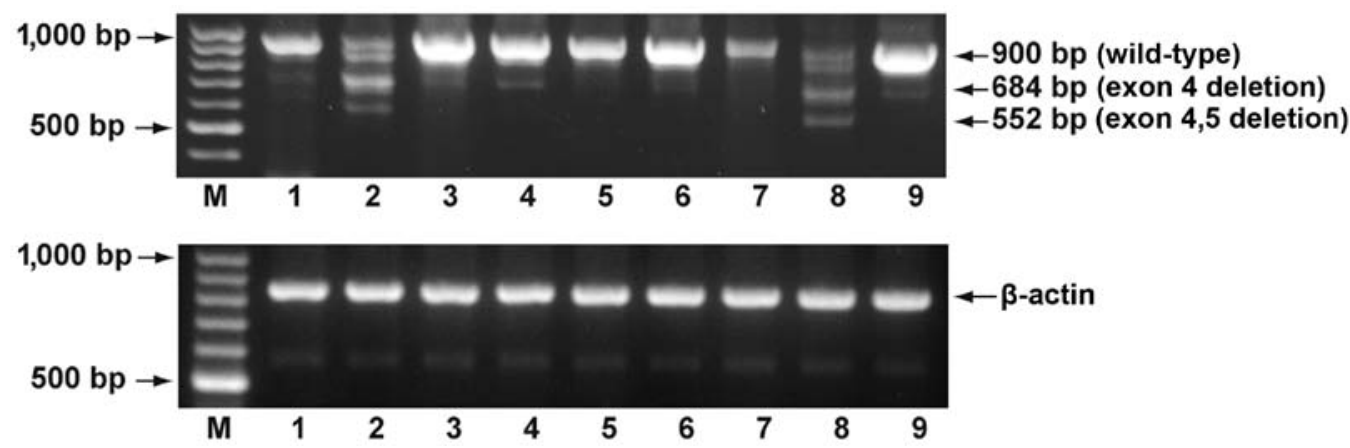

Figure 2. Expression of regucalcin mRNA in various brain tissues. The coding region of the regucalcin gene was amplified using cDNA for total RNA obtained from human normal brain tissues with the method of PCR. The amplified PCR products were visualized on a $1.8 \%$ agarose gel containing ethidium bromide. Data are representative of one of 3 experiments. M, 100 base DNA-ladder; lane 1, amygdala; lane 2, cerebral cortex; lane 3, cerebellum; lane 4, diencephalon; lane 5, hippocampus; lane 6 , medulla oblongata; lane 7 , optic nerve; lane 8 , pituitary; lane 9 , thalamus.

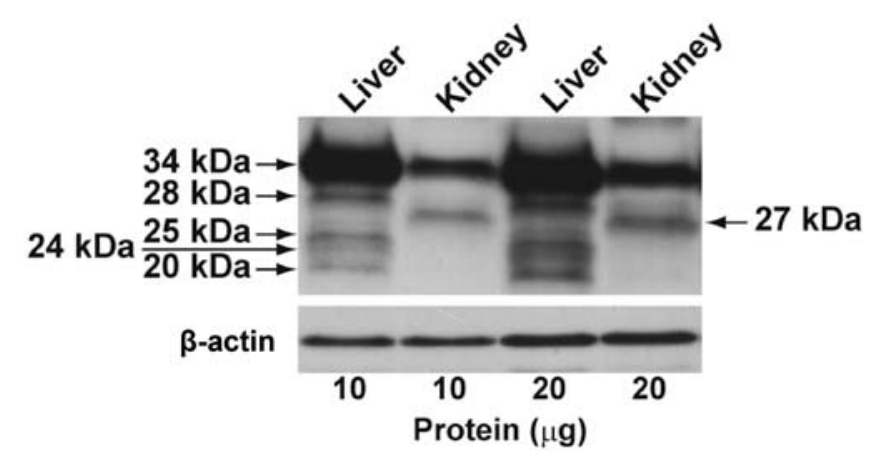

Figure 3. Expression of regucalcin protein in human normal liver and kidney tissues. Total protein lysates obtained from human normal liver and kidney tissues were subjected to SDS-PAGE and transferred electrophoretically to nitrocellulose membranes. Data are representative of one of 3 experiments. The blots were then incubated with regucalcin antibody overnight at $4^{\circ} \mathrm{C}$, followed by horseradish peroxidase-conjugated goat anti-rabbit IgG polyclonal antibodies for $1 \mathrm{~h}$ at room temperature and detected by enhanced chemiluminescence method.

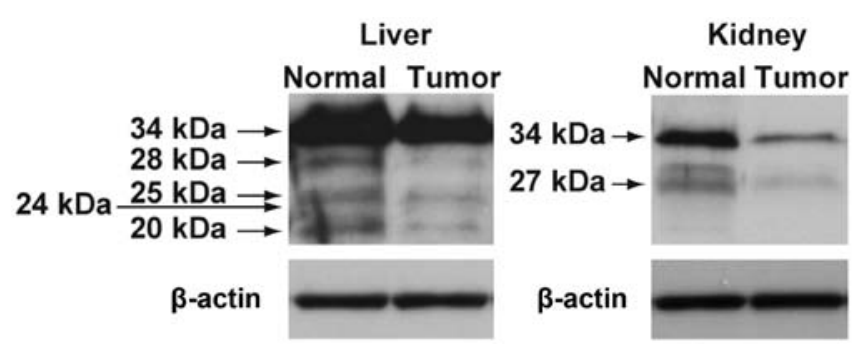

Figure 4. Regucalcin protein in human tumor liver and kidney tissues. The total protein lysates obtained from the normal and tumor (hepatocellular carcinoma and kidney clear cell carcinoma) tissues of human liver and kidney were applied for western blot analysis as described in Materials and methods. Data are representative of one of 3 experiments.

tumor tissues of liver hepatocellular carcinoma and kidney clear cell carcinoma as compared with those of the normal tissues.

Alternatively spliced variants for regucalcin mRNA and its related proteins (including 28, 25, 24 and $20 \mathrm{kDa}$ ) were not observed in the liver and kidney of rats and mice (data not shown).

\section{Discussion}

This study demonstrates that regucalcin mRNA expression is suppressed in various human tumor tissues, including hepatocellular carcinoma, kidney transitional cell carcinoma, brain malignant meningioma and lung non-small cell carcinoma evaluated by the clinical diagnosis of human subjects. Regucalcin protein was clearly decreased in the liver and kidney tumor tissues, although its levels were not determined in the brain and lung tumor tissues. Regucalcin has also been reported to be underexpressed in human hepatocellular carcinoma (26), breast and prostate cancers (27).

It has been reported that several transcripts of regucalcin mRNA are present in human breast (MCF-7) and prostate (LNCap) cancer cell lines (27). In this study, we found 3 transcripts for regucalcin mRNA in the normal (non-tumor) and tumor tissues of human liver, kidney, brain and lung. Moreover, we identified 2 alternatively spliced variants lacking exon 4 or exons 4 plus 5 of the human regucalcin gene. The identified alternatively spliced variants encoded 2 predicted proteins of 227 amino acid residues $(\sim 25 \mathrm{kDa})$ and 183 amino acid residues $(\sim 20 \mathrm{kDa})$. This observation on the alternative splicing was in accordance with previous findings on non-neoplastic human breast and prostate tissues and human breast (MCF-7) and prostate (LNCap) cancer cells (27). We also found that the expression of the alternatively spliced regucalcin transcripts was decreased in the human tumor tissues. Accordingly, it is interesting whether the alternative splicing event of the regucalcin gene has a biological significance in the human normal (non-tumor) and tumor tissues.

Two alternatively spliced regucalcin transcripts [684 bp (exon 4 deletion) and 552 bp (exon 4 and 5 deletion)] were not found in the various tissues (including liver, kidney cortex, heart, brain and the others) of normal rat and mouse (data not shown), in accordance with previous studies $(5,9)$. However, the alternatively spliced variants of the regucalcin gene were found in various human tissues. This physiological significance in the appearance of alternatively spliced variants for the regucalcin gene expression in human tissues is unknown. 
It is possible, however, that these variants play a role in the physiological function of regucalcin in human tissues.

The characterization of regucalcin gene expression in hepatoma cells is poorly understood. The mRNA expression, post-translational processing and targeting of the gene may be frequently altered in transformed cells. Regucalcin mRNA expression in tumor tissues have been shown to be decreased as compared with that in non-tumor tissues of chemically-fed rats, while regucalcin mRNA expression has been observed in transplantable Morris hepatoma cells (20). The sequencing of cDNA cloning for regucalcin in human liver tissues and cloned human hepatoma (HepG2) cells has been previously compared (28). We found that the human gene for regucalcin gives rise to transcripts with different 5'-UTR sequences in hepatoma cells; these genes contain identical coding regions, differing only in their untranslated sequences. Moreover, northern blot analysis using poly $(\mathrm{A})^{+}$RNA from human liver and HepG2 cells revealed that regucalcin mRNA in the HepG2 cells was longer than that of the liver (28). These observations demonstrate the existence of transcript heterogeneity of the human gene for regucalcin in cancer cells. Regucalcin mRNA expression has been shown to be decreased in HepG2 cells (30), and this reduction may be implicated in the transcriptional alteration (30). From these findings, it can be hypothesized that the suppressed regucalcin gene expression in tumor tissues plays a role in transcriptional alteration.

We also examined the expression of regucalcin protein in the liver and kidneys using normal human tissues. Full-length regucalcin protein $(34 \mathrm{kDa})$ was expressed in the human normal liver and kidney tissues. The regucalcin splice variant proteins (25 and $20 \mathrm{kDa}$ ) were found in the normal liver, but not in the normal kidney. As regucalcin splice variant proteins were not found in the normal kidney, their expression may be tissue specific. It has been reported that the proteolytic processing of full-length regucalcin protein $(34 \mathrm{kDa})$ leads to the production of 2 molecular weight proteins of 28 and $24 \mathrm{kDa}(29)$. In our study, the liver proteins of 28 or $24 \mathrm{kDa}$ and the kidney protein of $27 \mathrm{kDa}$, which did not correspond to the predicted molecular weight of the alternatively spliced variant proteins, were detected at minimal levels. These proteins were specifically observed in each tissue and appeared to be produced by proteolysis. It is unknown whether these proteins have a functional role.

Furthermore, we found that the expression of full-length regucalcin protein is suppressed in human liver hepatocellular carcinoma and kidney clear cell carcinoma. Regucalcin mRNA expression and its protein levels have been shown to be decreased in cloned rat hepatoma H4-II-E cells as compared to those of normal rat liver $(24,25)$. The overexpression of endogenous regucalcin has been found to suppress the proliferation of cloned rat hepatoma H4-II-E cells enhanced with serum stimulation $(17,24,25)$. Regucalcin has been demonstrated to cause $\mathrm{G} 1$ and $\mathrm{G} 2 / \mathrm{M}$ phase cell cycle arrest in H4-II-E cells (25). In addition, regucalcin has been shown to have suppressive effects on cell proliferation, inducing G1 and G2/M phase cell cycle arrest in cloned normal rat kidney proximal tubular epithelial NRK52E cells (31). The suppressive effects of regucalcin on cell proliferation may be mediated through depression in various $\mathrm{Ca}^{2+}$ signaling-dependent protein kinases, protein phosphatases and PI3 kinase activities and the suppression of c-myc, Ha-ras, $c$-jun and chk2 mRNA expression or the enhancement of $p 53$ and $R b$ mRNA expression $(24,25,32)$. Moreover, regucalcin has been shown to suppress protein synthesis and nuclear DNA and RNA synthesis $(16,17,19)$. Suppressed regucalcin gene expression may lead to the development of carcinogenesis $(16,21)$.

In conclusion, our study demonstrates that the full-length and alternatively spliced variants of regucalcin mRNA were expressed in various normal human tissues, including the liver, kidneys, brain and lungs, and that the expression of full-length regucalcin protein was found in human normal liver and kidney tissues. We also found that the spliced variant proteins of regucalcin appeared in the human normal liver but not in the kidney tissues.

\section{References}

1. Yamaguchi $\mathrm{M}$ and Yamamoto T: Purification of calcium binding substance from soluble fraction of normal rat liver. Chem Pharm Bull (Tokyo) 26: 1915-1918, 1978.

2. Yamaguchi M: A novel $\mathrm{Ca}^{2+}$-binding protein regucalcin and calcium inhibition. Regulatory role in liver cell function. In: Calcium Inhibition. Kohama K (ed). Japan Sci Soc Press, Tokyo and CRC Press, Boca Raton, pp.19-41, 1992.

3. Shimokawa $\mathrm{N}$ and Yamaguchi M: Molecular cloning and sequencing of the cDNA coding for a calcium-binding protein regucalcin from rat liver. FEBS Lett 327: 251-255, 1993.

4. Misawa $\mathrm{H}$ and Yamaguchi $\mathrm{M}$ : The gene of $\mathrm{Ca}^{2+}$-binding protein regucalcin is highly conserved in vertebrate species. Int $\mathrm{J}$ Mol Med 6: 191-196, 2000.

5. Yamaguchi M: The transcriptional regulation of regucalcin gene expression. Mol Cell Biochem 346: 147-171, 2011.

6. Shimokawa N, Matsuda Y and Yamaguchi M: Genomic cloning and chromosomal assignment of rat regucalcin gene. Mol Cell Biochem 151: 157-163, 1995.

7. Thiselton DL, McDowall J, Brandau O, Ramser J, d'Esposito F, Bhattacharga SS, Ross MT, Hardcastle AJ and Meindl M: An integrated, functionally annotated gene map of the DXS8026-ELK1 internal on human Xp11.3-Xp11.23: Potential hotspot for neurogenetic disorders. Genomics 79: 560-572, 2002.

8. Yamaguchi M, Makino R and Shimokawa N: The 5'end seguences and exon organization in rat regucalcin gene. Mol Cell Biochem 165: 145-150, 1996.

9. Shimokawa $\mathrm{N}$ and Yamaguchi $\mathrm{M}$ : Calcium administration stimulates the expression of calcium-binding protein regucalcin mRNA in rat liver. FEBS Lett 305: 151-154, 1992.

10. Yamaguchi $M$ and Isogai $M$ : Tissue concentration of calcium-binding protein regucalcin in rats by enzyme-linked immunoadsorbent assay. Mol Cell Biochem 122: 65-68, 1993.

11. Yamaguchi M: Hormonal regulation of regucalcin gene expression: Involvement in cell metabolism. Horm Stud 1: 1, 2013.

12. Yamaguchi M: Role of regucalcin in calcium signaling. Life Sci 66: 1769-1780, 2000

13. Yamaguchi M: Role of regucalcin in maintaining cell homeostasis and function. Int J Mol Med 15: 372-389, 2005.

14. Yamaguchi M: Regucalcin and cell regulation: role as a suppressor in cell signaling. Mol Cell Biochem 353: 101-137, 2011.

15. Yamaguchi M: Role of regucalcin in cell nuclear regulation: Involvement as a transcription factor. Cell Tissue Res 354: 331-341, 2013.

16. Yamaguchi M: Suppressive role of regucalcin in liver cell proliferation: Involvement in carcinogenesis. Cell Prolif 46: 243-253, 2013.

17. Yamaguchi M: The anti-apoptotic effect of regucalcin is mediated through multisignaling pathways. Apoptosis 18: 1145-1153, 2013.

18. Yamaguchi M: Regucalcin and metabolic disorder: osteoporosis and hyperlipidemia are induced in regucalcin transgenic rats. Mol Cell Biochem 327: 53-63, 2010.

19. Yamaguchi $\mathrm{M}$ and Murata $\mathrm{T}$ : Involvement of regucalcin in lipid metabolism and diabetes. Metabolism 62: 1045-1051, 2013.

20. Makino R and Yamaguchi M: Expression of calcium-binding protein regucalcin mRNA in hepatoma cells. Mol Cell Biochem 155: 85-90, 1996. 
21. Suzuki S, Asamoto M, Tsujimura K and Shirai T: Specific differences in gene expression profile revealed by cDNA microarray analysis of glutathione S-transferase placental form (GST-P) immunohistochemically positive rat liver foci and surrounding tissue. Carcinogenesis 25: 439-443, 2004.

22. Inagaki S and Yamaguchi M: Regulatory role of endogenous regucalcin in the enhancement of nuclear deoxyribonucleic acid synthesis with proliferation of cloned rat hepatoma cells (H4-II-E). J Cell Biochem 82: 704-711, 2001.

23. Misawa H, Inagaki S and Yamaguchi M: Suppression of cell proliferation and deoxyribonucleic acid synthesis in cloned rat hepatoma H4-II-E cells overexpressing regucalcin. J Cell Biochem 84: 143-149, 2002.

24. Tsurusaki Y and Yamaguchi M: Overexpression of regucalcin modulates tumor-related gene expression in cloned rat hepatoma H4-II-E cells. J Cell Biochem 90: 619-626, 2003.

25. Yamaguchi $M$ and Daimon Y: Overexpression of regucalcin suppresses cell proliferation in cloned rat hepatoma H4-II-E cells: Involvement of intracellular signaling factors and cell cycle-related genes. J Cell Biochem 95: 1169-1177, 2005.

26. Zhou SF, Mo FR, Bin YH, Hou GQ, Xie XX and Luo GR: Serum immunoreactivity of SMP30 and its tissues expression in hepatocellular carcinoma. Clin Biochem 44: 331-336, 2011.
27. Maia C, Santos C, Schmitt F and Socorro S: Regucalcin is under-expressed in human breast and prostate cancers: Effect of sex steroid hormones. J Cell Biochem 107: 667-676, 2009.

28. Misawa $\mathrm{H}$ and Yamaguchi M: Transcript heterogeneity of the human gene for $\mathrm{Ca}^{2+}$-binding protein regucalcin. Int $\mathrm{J}$ Mol Med 5: 283-287, 2000.

29. Arun P, Aleti V,Parikh K, Manne V and Chilukuri N: Senescence marker protein 30 (SMP30) expression in eukaryotic cells: existence of multiple species and membrane localization. PLoS One 6: e16545, 2011.

30. Murata T, Synya N and Yamaguchi M: Expression of calcium-binding protein regucalcin $\mathrm{mRNA}$ in the cloned human hepatoma cells (HepG2): stimulation by insulin. Mol Cell Biochem 175: 163-168, 1997.

31. Nakagawa T, Sawada N and Yamaguchi M: Overexpression of regucalcin suppresses cell proliferation of cloned normal rat kidney proximal tubular epithelial NRK52E cells. Int J Mol Med 16: 637-643, 2005.

32. Tsurusaki Y and Yamaguchi M: Role of regucalcin in liver nuclear function: Binding of regucalcin to nuclear protein or DNA and modulation of tumor-related gene expression. Int J Mol Med 14: 277-281, 2004. 